TITLE:

\title{
A Finite-Difference Time-Domain Technique for Nonlinear Elastic Media and Its Application to Nonlinear Lamb Wave Propagation
}

\author{
AUTHOR(S):
}

Matsuda, Naoki; Biwa, Shiro

\section{CITATION:}

Matsuda, Naoki ... [et al]. A Finite-Difference Time-Domain Technique for Nonlinear Elastic Media and Its Application to Nonlinear Lamb Wave Propagation. Japanese Journal of Applied Physics 2012, 51(7S): 07GB14.

ISSUE DATE:

2012-07-20

URL:

http://hdl.handle.net/2433/194153

\section{RIGHT:}

(C) 2012 The Japan Society of Applied Physics; This is not the published version. Please cite only the published version.; この論文は出版社版で ありません。引用の際には出版社版をご確認ご利用ください。 
This article appeared as:

N. Matsuda and S. Biwa, A finite-difference time-domain technique for nonlinear elastic media and its application to nonlinear Lamb wave propagation, Japanese Journal of Applied Physics, Vol. 51 (2012), $07 \mathrm{~GB} 14$.

\title{
A finite-difference time-domain technique for nonlinear elastic media and its application to nonlinear Lamb wave propagation
}

\begin{abstract}
Naoki Matsuda and Shiro Biwa*
Department of Aeronautics and Astronautics, Graduate School of Engineering, Kyoto University, Katsura, Nishikyo-ku, Kyoto 615-8540, Japan

Abstract

A finite-difference time-domain technique for nonlinear elastic media is proposed, which can be applied to analyze finite amplitude elastic waves in solids. The kinematic and the material nonlinearities are considered, employing a general expression for the strain energy of an isotropic solid containing the second- and third-order terms of the strain components. The accuracy of the proposed technique is demonstrated by comparison with the analytical solution for the plane longitudinal wave propagation with finite amplitude. Two-dimensional simulations are performed to demonstrate the effectiveness of this formulation for Lamb waves. First, numerical simulations without the nonlinear effects are carried out, and the spectral peaks obtained from the calculated waveforms are shown to agree well with the theoretical dispersion curves of Lamb waves. As an example with the nonlinear effects, the harmonic generation in Lamb wave propagation is also demonstrated. The results show that the growth of the second-harmonic mode occurs for an incident-wave frequency selected in accordance with the analytical phase matching condition.
\end{abstract}

* Corresponding author: Shiro Biwa, E-mail biwa@kuaero.kyoto-u.ac.jp 


\section{Introduction}

There have recently been a number of studies of elastodynamic problems using the finite-difference time-domain (FDTD) method $^{1-6)}$. This method is also referred to as the velocity-stress finite-difference or staggered grid finite-difference formulation ${ }^{7}$, where Navier's equations are decomposed to a set of first-order partial differential equations with respect to velocity and stress. The staggered grid finite-difference formulation has practical advantage for its stability and accuracy ${ }^{8}$. Most of previous studies of the FDTD simulation, however, assumed linear elastic bodies. Recent research has demonstrated that nonlinear features of ultrasonic waves can be used to evaluate the material degradation sensitively ${ }^{9-12}$. In this regard, it is important to gain understanding of nonlinear response in ultrasonic wave propagation. Especially, numerical methods are indispensable for the analysis of guided wave modes such as Lamb waves which are characterized by frequency-dispersion and multiple-mode existence.

In the present study, a formulation to deal with finite amplitude waves based on the FDTD method is presented. The kinematic as well as the material nonlinearities are considered in this formulation, employing a general expression for the strain energy of an isotropic solid containing the second- and third-order terms of the strain components.

Some results of numerical simulation applied to Lamb waves are shown based on this formulation. The dispersion curves constructed by the numerical results are compared to the analytical ones given by the Rayleigh-Lamb frequency equations ${ }^{13)}$. Furthermore, in the situation where a condition ${ }^{14)}$ of phase matching of fundamental and harmonic Lamb modes holds, cumulative harmonic generation is demonstrated as one of nonlinear effects in Lamb waves.

\section{Fundamental Equations of Nonlinear Elastodynamics}

To deal with finite amplitude waves, two sources of nonlinearity should be taken into account: the material nonlinearity and the kinematic nonlinearity. Accounting for the contribution of the terms which are cubic in the strains, the strain energy density $W$ is given by

$$
\rho_{0} W=\frac{1}{2} C_{i j k l} E_{i j} E_{k l}+\frac{1}{6} C_{i j k l m n} E_{i j} E_{k l} E_{m n},
$$

where $\rho_{0}$ is the mass density in the unstressed configuration, $E_{i j}$ are the components of the Lagrangian strain tensor, $E_{i j}=1 / 2\left(\partial u_{i} / \partial X_{j}+\partial u_{j} / \partial X_{i}+\partial u_{k} / \partial X_{i} \partial u_{k} / \partial X_{j}\right)$, and $C_{i j k l}$ and $C_{i j k l m n}$ are the components of the second- and the third-order stiffness tensors, respectively. The equations of motion, the displacement-velocity relation, and the stress-strain relation can be written by ${ }^{15}$

$$
\begin{gathered}
\rho_{0} \frac{\partial v_{i}}{\partial t}=\frac{\partial P_{i j}}{\partial X_{j}}, \\
\frac{\partial u_{i}}{\partial t}=v_{i}, \\
P_{i j}=C_{i j k l} \frac{\partial u_{k}}{\partial X_{l}}+\frac{1}{2}\left(C_{i j k l m n}+C_{i j l n} \delta_{k m}+C_{j n k l} \delta_{i m}+C_{j l m n} \delta_{i k}\right) \frac{\partial u_{k}}{\partial X_{l}} \frac{\partial u_{m}}{\partial X_{n}},
\end{gathered}
$$

where $X_{i}$ are the Lagrangian (or material) coordinates, $v_{i}$ the velocities, $u_{i}$ the displacements and $P_{i j}$ the components of a non-symmetric tensor known as the first Piola-Kirchhoff stress tensor. When the solid is isotropic, the stiffness tensors in eqs. (1) and (4) are given ${ }^{15)}$ as

$$
C_{i j k l}=\lambda \delta_{i j} \delta_{k l}+2 \mu I_{i j k l} \text {, }
$$


(a)

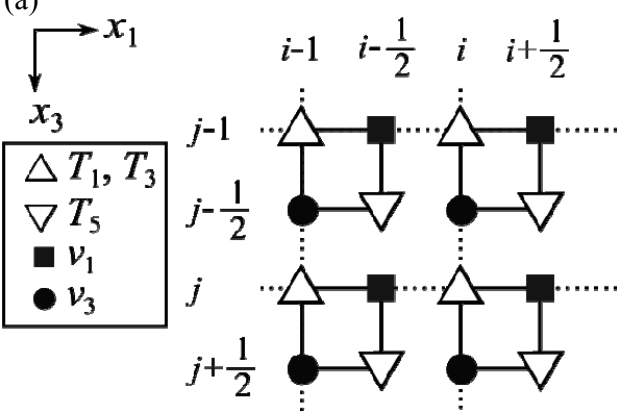

(b)

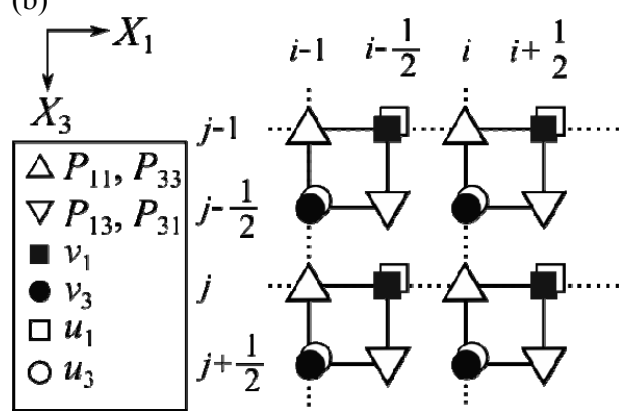

Fig. 1 Geometry of (a) the conventional FDTD grid and (b) the FDTD grid for nonlinear simulation.

$$
\begin{aligned}
C_{i j k l m n} & =\frac{A}{2}\left(\delta_{i k} I_{j l m n}+\delta_{i l} I_{j k m n}+\delta_{j k} I_{i l m n}+\delta_{j l} I_{i k m n}\right) \\
& +2 B\left(\delta_{i j} I_{k l m n}+\delta_{k l} I_{m n i j}+\delta_{m n} I_{i j k l}\right) \\
& +2 C \delta_{i j} \delta_{k l} \delta_{m n},
\end{aligned}
$$

where $\lambda$ and $\mu$ are the Lamé elastic constants and $A, B$ and $C$ are the third-order elastic constants in isotropic media used by Landau and Lifshitz ${ }^{16)}, \delta_{i k}$ is the Kronecker's delta and $I_{i j k l}=\left(\delta_{i k} \delta_{j l}+\delta_{i l} \delta_{j k}\right) / 2$.

\section{Formulation of FDTD Scheme for Nonlinear Elastic Media}

\subsection{Discretization}

In this section we introduce a two-dimensional FDTD scheme for finite amplitude elastic waves. Figure 1(a) shows the conventional FDTD staggered grid, where the nodes for velocity and stress are only considered. For the nonlinear problems considered in this paper, the time derivative of the stress cannot be expressed only by the velocity gradients as in the conventional FDTD formulation. For this reason, the nodes of the particle displacements are also required in the grid for nonlinear simulations as shown in Fig. 1(b). This grid corresponds to the $X_{1}-X_{3}$ plane of the material coordinates.

Also, the nodes of the stress tensor in the conventional staggered grid should be replaced with those of the first Piola-Kirchhoff stress tensor in this grid. Since the first Piola-Kirchhoff stress tensor is generally not symmetric, $P_{13}$ and $P_{31}$ are stored separately. Figure 1(b) shows the schematic of the staggered grid for nonlinear simulations.

The stresses and the velocities in the conventional FDTD scheme are alternately updated. In the FDTD scheme with nonlinear effects, the displacements and stresses $\left(u_{1}, u_{3}, P_{11}, P_{33}, P_{13}, P_{31}\right)$ are updated when the time step index is integer and the velocities $\left(v_{1}, v_{3}\right)$ are updated when it is half-integer. The updated displacements are given as follows by discretizing eq. (3):

$$
u_{1}{ }^{n}\left(i+\frac{1}{2}, j\right)=u_{1}{ }^{n-1}\left(i+\frac{1}{2}, j\right)+\Delta t v_{1}^{n-1 / 2}\left(i+\frac{1}{2}, j\right)
$$




$$
u_{3}{ }^{n}\left(i, j+\frac{1}{2}\right)=u_{3}{ }^{n-1}\left(i, j+\frac{1}{2}\right)+\Delta t v_{3}{ }^{n-1 / 2}\left(i, j+\frac{1}{2}\right),
$$

where $i$ and $j$ are the indices of a grid point in the $X_{1}$ and $X_{3}$ direction, respectively. The superscript index $n$ denotes the time step and $\Delta t$ is its increment. By substituting the updated displacements to eq. (4), the components of the first Piola-Kirchhoff stress tensor are given by

$$
\begin{aligned}
& P_{11}{ }^{n}(i, j)=c_{11} u_{1,1}{ }^{n}(i, j)+c_{13} u_{3,3}{ }^{n}(i, j) \\
& +d_{1}\left[u_{1,1}{ }^{n}(i, j)\right]^{2} \\
& +d_{2}\left[2 u_{1,1}{ }^{n}(i, j)+u_{3,3}{ }^{n}(i, j)\right] u_{3,3}{ }^{n}(i, j) \\
& +d_{3}\left\{\left[u_{1,3}{ }^{n}(i, j)\right]^{2}+\left[u_{3,1}{ }^{n}(i, j)\right]^{2}\right\} \\
& +d_{4} u_{1,3}{ }^{n}(i, j) u_{3,1}{ }^{n}(i, j), \\
& P_{33}{ }^{n}(i, j)=c_{11} u_{3,3}{ }^{n}(i, j)+c_{13} u_{1,1}{ }^{n}(i, j) \\
& +d_{1}\left[u_{3,3}{ }^{n}(i, j)\right]^{2} \\
& +d_{2}\left[u_{1,1}{ }^{n}(i, j)+2 u_{3,3}{ }^{n}(i, j)\right] u_{1,1}{ }^{n}(i, j) \\
& +d_{3}\left\{\left[u_{1,3}{ }^{n}(i, j)\right]^{2}+\left[u_{3,1}{ }^{n}(i, j)\right]^{2}\right\} \\
& +d_{4} u_{1,3}{ }^{n}(i, j) u_{3,1}{ }^{n}(i, j), \\
& P_{13}{ }^{n}\left(i+\frac{1}{2}, j+\frac{1}{2}\right)=c_{55}\left[u_{1,3}{ }^{n}\left(i+\frac{1}{2}, j+\frac{1}{2}\right)+u_{3,1}{ }^{n}\left(i+\frac{1}{2}, j+\frac{1}{2}\right)\right] \\
& +\left[u_{1,1}{ }^{n}\left(i+\frac{1}{2}, j+\frac{1}{2}\right)+u_{3,3}{ }^{n}\left(i+\frac{1}{2}, j+\frac{1}{2}\right)\right] \\
& \times\left[2 d_{3} u_{1,3}{ }^{n}\left(i+\frac{1}{2}, j+\frac{1}{2}\right)+d_{4} u_{3,1}{ }^{n}\left(i+\frac{1}{2}, j+\frac{1}{2}\right)\right], \\
& P_{31}{ }^{n}\left(i+\frac{1}{2}, j+\frac{1}{2}\right)=c_{55}\left[u_{1,3}{ }^{n}\left(i+\frac{1}{2}, j+\frac{1}{2}\right)+u_{3,1}{ }^{n}\left(i+\frac{1}{2}, j+\frac{1}{2}\right)\right] \\
& +\left[u_{1,1}{ }^{n}\left(i+\frac{1}{2}, j+\frac{1}{2}\right)+u_{3,3}{ }^{n}\left(i+\frac{1}{2}, j+\frac{1}{2}\right)\right] \\
& \times\left[2 d_{3} u_{3,1}{ }^{n}\left(i+\frac{1}{2}, j+\frac{1}{2}\right)+d_{4} u_{1,3}{ }^{n}\left(i+\frac{1}{2}, j+\frac{1}{2}\right)\right],
\end{aligned}
$$

where commas are used to denote the partial derivatives with respect to the material coordinates, whose discretized forms are defined in eqs. (A·1) and (A-2) in the Appendix. The coefficients $c_{11}, c_{13}$, $c_{55}, d_{1}, d_{2}, d_{3}$, and $d_{4}$ are the linear combinations of the second- or third-elastic constants in isotropic media defined as follows: 


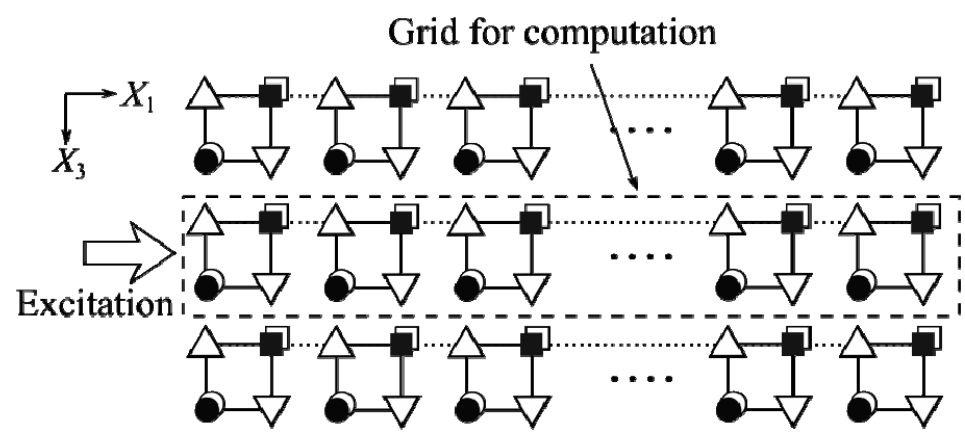

Fig. 2 Schematic of the grid for plane longitudinal wave simulation.

$$
\begin{aligned}
& c_{11}=\lambda+2 \mu, \\
& c_{13}=\lambda, \\
& c_{55}=\mu, \\
& d_{1}=\frac{3}{2} \lambda+3 \mu+A+3 B+C, \\
& d_{2}=\frac{1}{2} \lambda+B+C, \\
& d_{3}=\frac{1}{2} \lambda+\mu+\frac{1}{4} A+\frac{1}{2} B, \\
& d_{4}=\mu+\frac{1}{2} A+B .
\end{aligned}
$$

Equation (2) gives the velocities of the next time step:

$$
\begin{aligned}
& v_{1}^{n+1 / 2}\left(i+\frac{1}{2}, j\right)=v_{1}{ }^{n-1 / 2}\left(i+\frac{1}{2}, j\right)+\frac{\Delta t}{\rho_{0}}\left[P_{11,1}{ }^{n}\left(i+\frac{1}{2}, j\right)+P_{13,3}{ }^{n}\left(i+\frac{1}{2}, j\right)\right], \\
& v_{3}^{n+1 / 2}\left(i, j+\frac{1}{2}\right)=v_{3}{ }^{n-1 / 2}\left(i, j+\frac{1}{2}\right)+\frac{\Delta t}{\rho_{0}}\left[P_{31,1}{ }^{n}\left(i, j+\frac{1}{2}\right)+P_{33,3}{ }^{n}\left(i, j+\frac{1}{2}\right)\right],
\end{aligned}
$$

where the spatial gradients of the first Piola-Kirchhoff stress tensor are defined in the Appendix [eq. (A-3)]. Using eqs. (7)-(12), (14), and (15), we alternately calculate the stresses and velocities according to when the time step index is integer or half-integer, respectively.

The above discretization procedure applies to nodes in the interior of the elastic solid. At the boundaries, the computation of the stress components requires different expressions of the displacement gradients from those for the interior points given in the Appendix. Namely, at the boundaries the central finite-differences are replaced by certain forward (or backward) finite-differences using the displacements of interior nodes.

\subsection{Verification of the accuracy of nonlinear simulation}

To confirm the accuracy of the proposed FDTD scheme for nonlinear elastic media, we demonstrate a nonlinear simulation of plane longitudinal wave propagation in aluminum. Due to the one-dimensional nature of the problem, each variable is computed at $20,000 \times 1$ grid points, and the same numerical values are assigned to the corresponding grid points located one-grid above and below (Fig. 2). The longitudinal and transverse wave speeds of aluminum are $c_{\mathrm{L}}=6350 \mathrm{~m} / \mathrm{s}$ and 


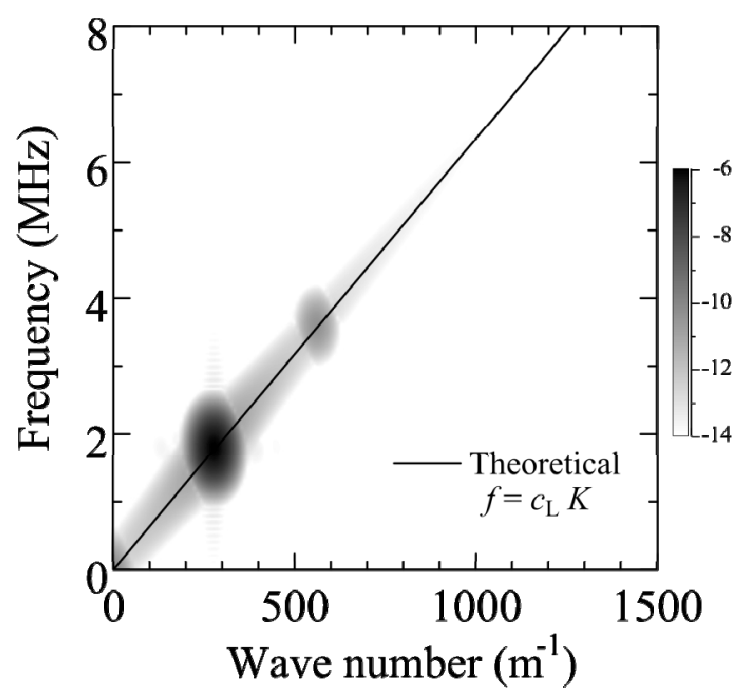

Fig. $3 f$ - $K$ distribution of the longitudinal wave simulation at the propagation distance of $88 \mathrm{~mm}$. The color is based on natural logarithm of the amplitude $U(K, f)$.

$c_{\mathrm{T}}=3130 \mathrm{~m} / \mathrm{s}$, respectively $\left(\lambda=56.0 \mathrm{GPa}, \mu=26.5 \mathrm{GPa}, \rho_{0}=2700 \mathrm{~kg} / \mathrm{m}^{3}\right)$, and their third-order elastic constants are $A=-2.96 \times 10^{11} \mathrm{~Pa}, B=-1.15 \times 10^{11} \mathrm{~Pa}$ and $C=9.35 \times 10^{10} \mathrm{~Pa}^{17)}$. The excitation is made by imposing a continuous sinusoidal displacement with the frequency $1.8 \mathrm{MHz}$ and the amplitude $10 \mathrm{~nm}$ to the left boundary. After collecting the computed displacement waveforms at different spatial locations, we obtain the displacement distribution $u_{1}\left(X_{1}, t\right)$. The displacement distribution is analyzed by two-dimensional Fourier transform in the frequency-wave number plot $U(K, f)$, where $K$ and $f$ are the wave number (inverse of wave length) and the frequency, respectively. The two-dimensional Fourier transform is calculated as

$$
U(K, f)=\int_{-\infty}^{\infty} \int_{-\infty}^{\infty} W_{\bar{X}_{1}, \bar{t}}\left(X_{1}, t\right) u\left(X_{1}, t\right) \mathrm{e}^{i 2 \pi\left(K X_{1}-f t\right)} d X_{1} d t,
$$

where $W_{\bar{X}_{1}, \bar{t}}\left(X_{1}, t\right)$ is the following window function centered at $X_{1}=\bar{X}$ and $t=\bar{t}$,

$$
W_{\bar{X}_{1}, \bar{t}}\left(X_{1}, t\right)=\left\{\begin{array}{l}
\frac{1}{4}\left(1+\cos \frac{\pi\left(X_{1}-\bar{X}_{1}\right)}{X}\right)\left(1+\cos \frac{\pi(t-\bar{t})}{T}\right) \exp \left[-\left(\frac{3\left(X_{1}-\bar{X}_{1}\right)}{X}\right)^{2}-\left(\frac{3(t-\bar{t})}{T}\right)^{2}\right], \\
\quad-X<X_{1}-\bar{X}_{1}<X \text { and }-T<t-\bar{t}<T \\
0, \quad \text { otherwise. }
\end{array}\right.
$$

In this analysis, we choose $X=45 \mathrm{~mm}$ and $T=5 \mu \mathrm{s}$. Figure 3 shows a result of the two-dimensional Fourier transform analysis at $\bar{X}=88 \mathrm{~mm}, \bar{t}=26 \mu$ s. Because the temporal and spatial widths of the window function are finite, the peaks are not sharp but show some spread. We can observe the second harmonic $(3.6 \mathrm{MHz})$ generated from the fundamental wave $(1.8 \mathrm{MHz})$ in Fig. 3. The amplitude ratios of the second harmonic and the fundamental wave are shown in Fig. 4 as a function of the propagation distance $X_{1}$. The plotted points appear to align on a straight line. When fitted by a quadratic function which passes through the origin, the plots give the slope of $4.17 \times 10^{-4} \mathrm{~m}^{-1}$ at the origin.

A simple perturbation analysis ${ }^{15,18)}$ shows that the longitudinal wave excited by $u_{1}(0, t)=u_{0} \sin \omega t$ 


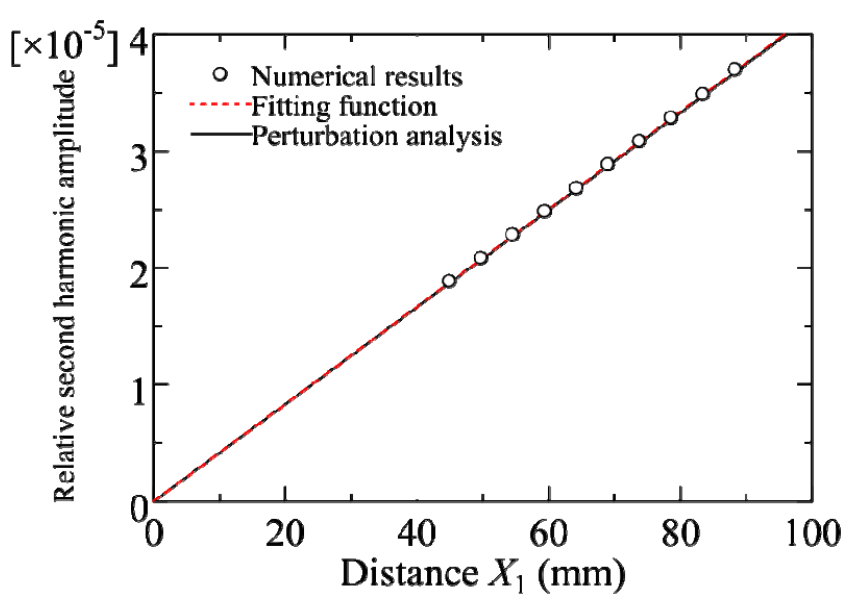

Fig. 4 (Color online) Variation of the relative amplitude of the second harmonics with the propagation distance. The plotted circles are the result of the numerical simulation. The solid line and the red dashed line are the perturbation solution and the fitted function of the numerical results, respectively.

produces the following wave containing the second harmonic component as

$$
\begin{gathered}
u_{1}\left(X_{1}, t\right)=u_{0} \sin \left(\omega t-k X_{1}\right)+\frac{\beta}{4}\left(\frac{\omega u_{0}}{c_{\mathrm{L}}}\right)^{2} X_{1} \cos 2\left(\omega t-k X_{1}\right), \\
\beta=-\left(\frac{3}{2}+\frac{2 A+6 B+2 C}{2 \rho_{0} c_{\mathrm{L}}^{2}}\right),
\end{gathered}
$$

where $u_{1}\left(X_{1}, t\right)$ denotes the particle displacement and $k=\omega / c_{\mathrm{L}}$. From eq. (18), the amplitude ratio of the second harmonic and the fundamental components $R\left(X_{1}\right)$ is proportional to the propagation distance $X_{1}$. The amplitude ratio $R\left(X_{1}\right)$ is written as

$$
R\left(X_{1}\right)=\frac{\beta u_{0} \omega^{2}}{4 c_{\mathrm{L}}^{2}} X_{1}=k_{0} X_{1},
$$

where $k_{0}=4.16 \times 10^{-4} \mathrm{~m}^{-1}$ in the case of the $1.8 \mathrm{MHz}$ longitudinal wave propagation in aluminum with the above-mentioned parameters. The difference of the spatial slope of the relative second harmonic amplitude obtained by the numerical simulation and by the perturbation analysis is only $0.3 \%$. Therefore, the proposed numerical simulation scheme has enough accuracy in respect of the amplitude of the second harmonic generation.

\section{Application to Lamb Wave Propagation}

Two examples of numerical simulations for Lamb wave propagation are demonstrated, one without nonlinear effects and one with nonlinear effects. Both examples are for a two-dimensional cross section of an aluminum plate ( $2 \mathrm{~mm}$ thick, $400 \mathrm{~mm}$ long). A schematic of the simulation model is displayed in Fig. 5. The grid size is $\Delta d=0.02 \mathrm{~mm}$ and the number of the grid points is $20000 \times 100$. The size of time step $\Delta t$ is $0.79 \mathrm{~ns}$, which satisfies the stability condition. ${ }^{3)}$ The incident wave is excited from one side of the plate by prescribing velocities and/or stresses. The zero-stress formulation $^{3,19)}$ is applied to three other surfaces which are assumed to be traction-free. 


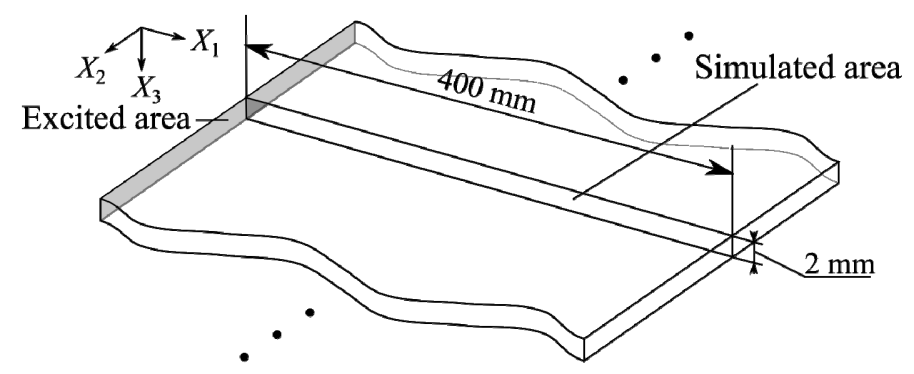

Fig. 5 The configuration of the model of Lamb wave simulation used for both linear and nonlinear simulations. The upper and the bottom sides of the plate are free of stress.

(a)

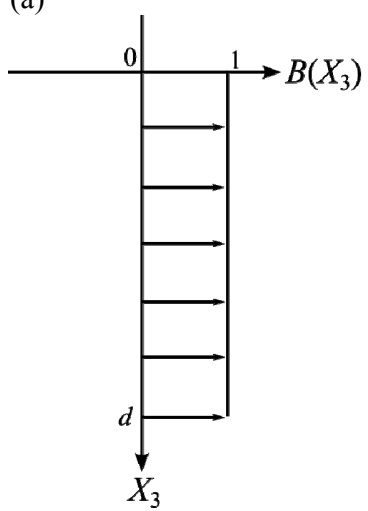

(b)

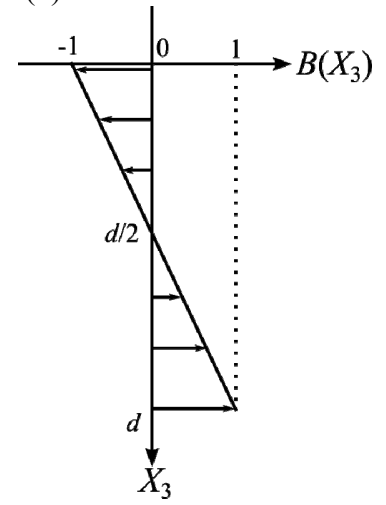

Fig. 6 Schematics of thickness profile of the excitation stress $B\left(X_{3}\right)$ where $d$ denotes the thickness of the plate, for the excitation of (a) symmetric and (b) antisymmetric Lamb waves.

\subsection{Example of linear simulation}

A linear simulation based on the above formulation, omitting the second-order terms of the spatial gradient of the particle displacements in eqs. (9) to (12), is performed to confirm the validity of the proposed technique. The excitation is given in terms of the stress $P_{11}$ at one surface of the model as shown in Fig. 5. The excitation stress with the amplitude $P_{0}$ is expressed as

$$
P_{11}=P_{0} A(t) B\left(X_{3}\right) \text {. }
$$

The temporal waveform $A(t)$ is a continuous sinusoidal wave which initially increases its amplitude as Gaussian function expressed as

$$
A(t)= \begin{cases}\exp \left[-\left(3 \frac{t-t_{\mathrm{c}}}{t_{\mathrm{c}}}\right)^{2}\right] \cos \left(2 \pi f_{0} t\right), & \left(0 \leq t<t_{\mathrm{c}}\right) \\ \cos \left(2 \pi f_{0} t\right), & \left(t_{\mathrm{c}} \leq t\right)\end{cases}
$$

where $t_{\mathrm{c}}=6 \mu \mathrm{s}$. In order to perform numerical simulations of symmetric and antisymmetric modes of Lamb waves separately, the thickness profile of the excitation stress $B\left(X_{3}\right)$ is given in the forms shown in Figs. 6(a) and 6(b). The simulation was performed by increasing the frequency $f_{0}$ of the incident wave from 0.5 to $5.0 \mathrm{MHz}$. The computed snapshots of $P_{11} / P_{0}$ at different time steps are shown in Fig. 7, for the antisymmetric-mode excitation at 3.0 MHz.

After collecting the computed surface displacement waveforms at different spatial locations, the results are analyzed by two-dimensional Fourier transform in the frequency-wave number $(f-K)$ plot. The plotted points in Fig. 8 denote the peaks of the $f-K$ plot at $\bar{X}=45 \mathrm{~mm}, \bar{t}=23 \mu$ s from the 


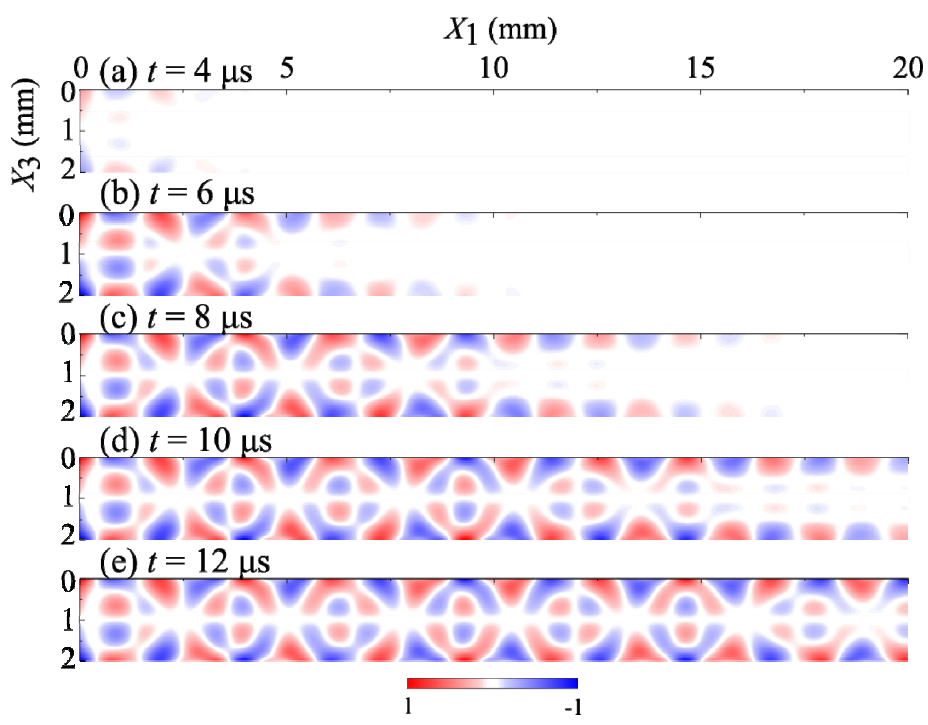

Fig. 7 (Color online) The computed snapshots of $P_{11} / P_{0}\left(0 \leq X_{1} \leq 20 \mathrm{~mm}\right)$ at the elapsed times (a) $4 \mu \mathrm{s}$, (b) 6 $\mu \mathrm{s}$, (c) $8 \mu \mathrm{s}$, (d) $10 \mu \mathrm{s}$, and (e) $12 \mu \mathrm{s}$.

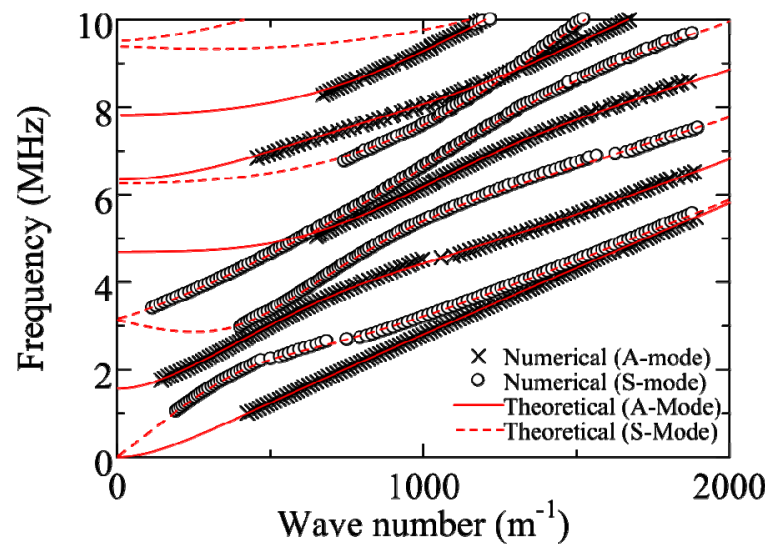

Fig. 8 (Color online) $f-K$ peaks of the numerical results and the analytical dispersion curves by the Rayleigh-Lamb equations. The plotted circles and crosses are the numerical results obtained by numerical simulation of symmetric and antisymmetric modes of Lamb waves, respectively. The solid and dashed lines are the theoretical dispersion curves given by the Rayleigh-Lamb frequency equations.

numerical simulation and the solid and dashed lines are the theoretical dispersion curves given by the Rayleigh-Lamb frequency equations. The plotted points generated by the numerical simulation show good agreement with the theoretical curves. The standard deviation of the difference between the numerical simulation and the theoretical dispersion curve is $1.4 \%$ in the wave number for given frequencies. This indicates the validity of the present FDTD technique.

\subsection{Example of nonlinear simulation}

While higher harmonics in dispersive Lamb waves do not generally grow with the propagation distance, it is known that certain special types of Lamb waves exhibit monotonic growth of the harmonics with the propagation distance. The fundamental wave and the second harmonic of theseLamb wave modes have identical phase velocity, and therefore the second harmonic Lamb 
(a)

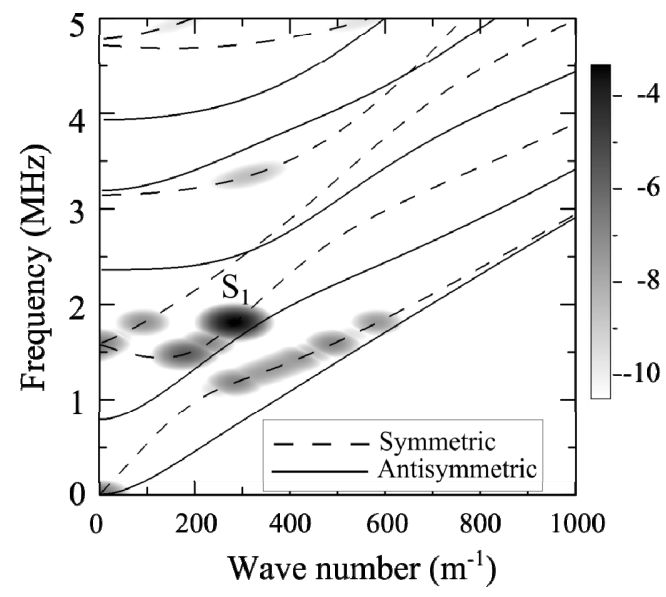

(b)

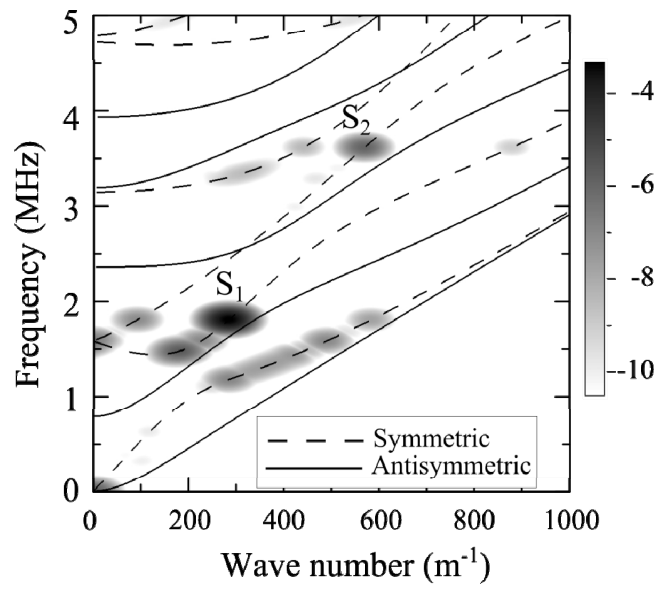

Fig. $9 f-K$ distribution by the numerical simulation at the propagation distance of $100 \mathrm{~mm}$, (a) without nonlinear effects and (b) with nonlinear effects. The color is based on natural logarithm of the amplitude of $U(K, f)$.

wave grows monotonically with the propagation distance as other elastic waves which have no dispersive nature ${ }^{20)}$. Recently the authors derived four types of the special Lamb modes ${ }^{14)}$ which are expected to generate higher harmonics in a cumulative manner. The four types are identified as (i) Lamé modes, (ii) symmetric modes with dominant longitudinal displacements, (iii) intersections of symmetric and antisymmetric modes and (iv) extra Rayleigh modes. As a specific example, the 1.8 $\mathrm{MHz}_{1}$-mode Lamb wave satisfies the condition for (ii) in an aluminum plate whose thickness is 2 $\mathrm{mm}$. Thereby we conducted numerical simulations when $\mathrm{S}_{1}$-mode Lamb wave was excited with the fundamental frequencies of 1.6, 1.8, and $2.0 \mathrm{MHz}$. The excitation is applied at one end of the plate in terms of both velocities and stresses with their thickness profiles given by an analytical Lamb wave solution at each frequency, with the incident temporal waveform expressed as eq. (22) with $t_{\mathrm{c}}$ also 6 $\mu \mathrm{s}$. The maximum amplitude of the thickness profile is chosen so that the energy flux of the excited Lamb mode is equal to that of the plane longitudinal wave with displacement amplitude $10 \mathrm{~nm}$. The simulation was performed with nonlinear terms. The third-order elastic constants are the same as those used in $\S 3.2$.

Figure 9(b) shows the result of the two-dimensional Fourier transform at $\bar{X}=100 \mathrm{~mm}, \bar{t}=$ $70 \mu \mathrm{s}$ when the incident wave frequency is $1.8 \mathrm{MHz}$. The corresponding result for the linear case is shown in Fig. 9(a), obtained with the same condition as Fig. 9(b) except that the coefficients $d_{1}, d_{2}$, $d_{3}$, and $d_{4}$ are switched to zeros. Solid and dashed lines in Fig. 9 denote the dispersion curves of the linear theory. In Fig. 9(b), we can observe the second harmonic $\left(\mathrm{S}_{2}\right.$ mode, 3.6 MHz) generated from the fundamental mode, which is not seen in Fig. 9(a). The amplitude ratio of the second harmonic and the fundamental wave was computed and shown in Fig. 10 as a function of the propagation distance from the excitation area. The relative amplitude of second harmonic from the $1.8 \mathrm{MHz}$ Lamb mode increases linearly as a function of the propagation distance, while those for other frequencies do not. This shows that the cumulative second harmonic generation of Lamb waves only occurs in special circumstances, and the present numerical analysis gives its occurrence at the theoretically predicted frequency. 


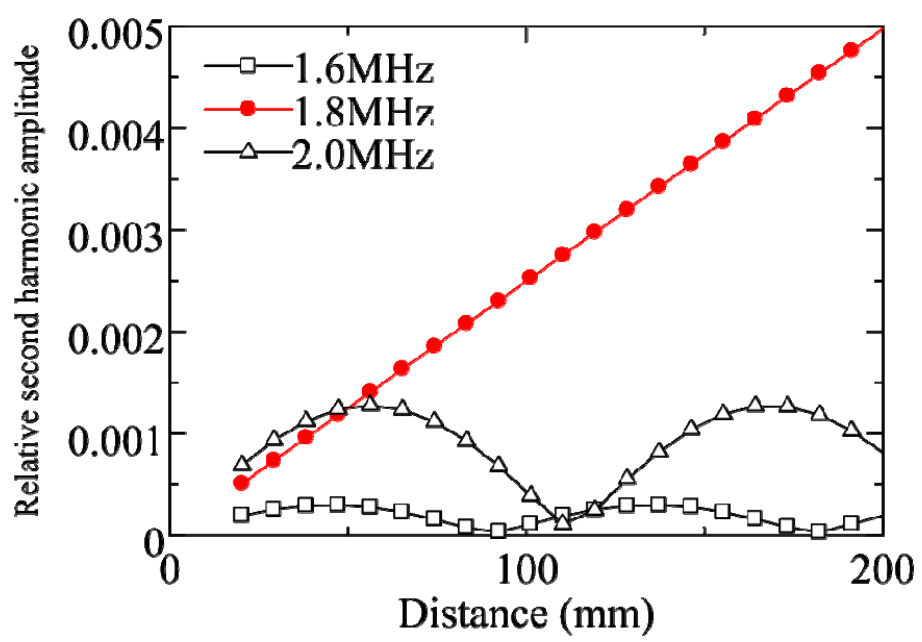

Fig. 10 (Color online) Variation of the relative amplitude of the second harmonics with the propagation distance.

\section{Conclusions}

A numerical formulation of the FDTD method for finite amplitude ultrasonic waves has been presented. The kinematic and the material nonlinearities are considered employing a general form of the strain energy with the second- and third-order terms of the strains. The simulated results of Lamb waves based on this formulation show good agreement with the theoretical dispersion curves. With the proposed technique, the cumulative second harmonic generation in Lamb waves can also be simulated.

\section{Appendix}

The discretized expressions for the spatial gradients of the displacements and the stresses are shown here, only for nodes in the interior of the solid. Because the positions of the nodes for $P_{11}$ and $P_{33}$ are different from those for $P_{13}$ and $P_{31}$ in the staggered grid (Fig. 1(b)), we must consider the spatial gradients of the displacements on both kinds of positions. The spatial gradients of the displacements $u_{1,1}, u_{3,3}, u_{1,3}$ and $u_{3,1}$ centered at $(i, j)$ are required to calculate $P_{11}$ and $P_{33}$, and those centered at $(i+1 / 2, j+1 / 2)$ are required to calculate $P_{13}$ and $P_{31}$. The gradients $u_{1,1}, u_{3,3}, u_{1,3}$ and $u_{3,1}$ centered at $(i, j)$ are expressed as 


$$
\begin{aligned}
u_{1,1}{ }^{n}(i, j)=\frac{1}{\Delta d} & {\left[u_{1}{ }^{n}\left(i+\frac{1}{2}, j\right)-u_{1}{ }^{n}\left(i-\frac{1}{2}, j\right)\right], } \\
u_{3,3}{ }^{n}(i, j)=\frac{1}{\Delta d} & {\left[u_{3}{ }^{n}\left(i, j+\frac{1}{2}\right)-u_{3}{ }^{n}\left(i, j-\frac{1}{2}\right)\right], } \\
u_{1,3}{ }^{n}(i, j)=\frac{1}{4 \Delta d} & {\left[u_{1}{ }^{n}\left(i+\frac{1}{2}, j+1\right)+u_{1}{ }^{n}\left(i-\frac{1}{2}, j+1\right)\right.} \\
- & \left.u_{1}{ }^{n}\left(i+\frac{1}{2}, j-1\right)-u_{1}{ }^{n}\left(i-\frac{1}{2}, j-1\right)\right], \\
u_{3,1}{ }^{n}(i, j)=\frac{1}{4 \Delta d} & {\left[u_{3}{ }^{n}\left(i+1, j+\frac{1}{2}\right)+u_{3}{ }^{n}\left(i+1, j-\frac{1}{2}\right)\right.} \\
- & \left.u_{3}{ }^{n}\left(i-1, j+\frac{1}{2}\right)-u_{3}{ }^{n}\left(i-1, j-\frac{1}{2}\right)\right],
\end{aligned}
$$

where $\Delta d$ denotes the grid size.

On the other hand, the gradients $u_{1,1}, u_{3,3}, u_{1,3}$ and $u_{3,1}$ centered at $(i+1 / 2, j+1 / 2)$ are expressed as

$$
\begin{aligned}
u_{1,1}{ }^{n}\left(i+\frac{1}{2}, j+\frac{1}{2}\right)=\frac{1}{4 \Delta d} & {\left[u_{1}{ }^{n}\left(i+\frac{3}{2}, j+1\right)+u_{1}{ }^{n}\left(i+\frac{3}{2}, j\right)\right.} \\
& \left.-u_{1}{ }^{n}\left(i-\frac{1}{2}, j+1\right)-u_{1}{ }^{n}\left(i-\frac{1}{2}, j\right)\right], \\
u_{3,3}{ }^{n}\left(i+\frac{1}{2}, j+\frac{1}{2}\right)=\frac{1}{4 \Delta d} & {\left[u_{3}{ }^{n}\left(i+1, j+\frac{3}{2}\right)+u_{3}{ }^{n}\left(i, j+\frac{3}{2}\right)\right.} \\
& \left.-u_{3}{ }^{n}\left(i+1, j-\frac{1}{2}\right)-u_{3}{ }^{n}\left(i, j-\frac{1}{2}\right)\right], \\
u_{1,3}{ }^{n}\left(i+\frac{1}{2}, j+\frac{1}{2}\right)= & \frac{1}{\Delta d}\left[u_{1}{ }^{n}\left(i+\frac{1}{2}, j+1\right)-u_{1}{ }^{n}\left(i+\frac{1}{2}, j\right)\right], \\
u_{3,1}{ }^{n}\left(i+\frac{1}{2}, j+\frac{1}{2}\right)= & \frac{1}{\Delta d}\left[u_{3}{ }^{n}\left(i+1, j+\frac{1}{2}\right)-u_{3}{ }^{n}\left(i, j+\frac{1}{2}\right)\right] .
\end{aligned}
$$

The spatial gradients of the first Piola-Kirchhoff tensor required in eqs. (14), (15) are discretized as

$$
\begin{aligned}
& P_{11,1}{ }^{n}\left(i+\frac{1}{2}, j\right)=\frac{1}{\Delta d}\left[P_{11}{ }^{n}(i+1, j)-P_{11}{ }^{n}(i, j)\right], \\
& P_{13,3}{ }^{n}\left(i+\frac{1}{2}, j\right)=\frac{1}{\Delta d}\left[P_{13}{ }^{n}\left(i+\frac{1}{2}, j+\frac{1}{2}\right)-P_{13}{ }^{n}\left(i+\frac{1}{2}, j-\frac{1}{2}\right)\right], \\
& P_{31,1}{ }^{n}\left(i, j+\frac{1}{2}\right)=\frac{1}{\Delta d}\left[P_{31}{ }^{n}\left(i+\frac{1}{2}, j+\frac{1}{2}\right)-P_{31}{ }^{n}\left(i-\frac{1}{2}, j+\frac{1}{2}\right)\right], \\
& P_{33,3}{ }^{n}\left(i, j+\frac{1}{2}\right)=\frac{1}{\Delta d}\left[P_{33}{ }^{n}(i, j+1)-P_{33}{ }^{n}(i, j)\right] .
\end{aligned}
$$




\section{References}

1) M. Sato: Jpn. J. Appl. Phys. 47 (2008) 3931.

2) Y. Nagatani, H. Imaizumi, T. Fukuda, M. Matsukawa, Y. Watanabe, and T. Otani: Jpn. J. Appl. Phys. 45 (2006) 7186.

3) R. W. Graves: Bull. Seismol. Soc. Am. 86 (1996) 1091.

4) H. Saito, J. Naoi, and T. Kikuchi: Jpn. J. Appl. Phys. 43 (2004) 3176.

5) K. Mori, T. Nakamura, T. Yokoyama, and A. Hasegawa: Jpn. J. Appl. Phys. 44 (2005) 4696.

6) Y. Shintaku, Y. Ohara, and K. Yamanaka: Jpn. J. Appl. Phys. 50 (2011) 096601.

7) J. Virieux: Geophysics 49 (1984) 1933.

8) A. Levander: Geophysics 53 (1988) 1425.

9) C. Pruell, J. Y. Kim, J. Qu, and L. J. Jacobs: Appl. Phys. Lett. 91 (2007) 231911.

10) J. H. Cantrell and W. T. Yost: Int. J. Fatigue 23 (2001) 487.

11) A. J. Croxford, P. D. Wilcox, B. W. Drinkwater, and P. B. Nagy: J. Acoust. Soc. Am. 126 (2009) 117.

12) Y. Xiang, M. Deng, F. Xuan, and C. Liu: Ultrasonics 51 (2011) 974.

13) K. F. Graff: Wave Motion in Elastic Solids (Dover, New York, 1975) Chap. 8, p. 437.

14) N. Matsuda and S. Biwa: J. Appl. Phys. 109 (2011) 094903.

15) A. N. Norris: in Nonlinear Acoustics, ed. M. Hamilton and D. Blackstock (Academic Press, San Diego, CA, 1998) p. 263.

16) L. D. Landau and E. M. Lifshitz: Theory of Elasticity (Elsevier, Amsterdam, 1986) 3rd ed., p. 106.

17) S. Kanemochi, M. Akaboshi, and K. Mizuno: Zairyo 27 (1978) 974 [in Japanese].

18) L. K. Zarembo and V. A. Krasil'nikov: Sov. Phys. Usp. 13 (1971) 778.

19) M. Sato: Acoust. Sci. Technol. 28 (2007) 49.

20) W. J. N. de Lima and M. Hamilton: J. Sound Vib. 265 (2003) 819. 